Théologiques

Théologiques

\title{
Mythe cosmogonique et dialogue scientifico-religieux
}

\section{Christian Downs}

Volume 9, numéro 1, printemps 2001

Les cosmologies

URI : https://id.erudit.org/iderudit/005685ar

DOI : https://doi.org/10.7202/005685ar

Aller au sommaire du numéro

\section{Éditeur(s)}

Faculté de théologie de l'Université de Montréal

\section{ISSN}

1188-7109 (imprimé)

1492-1413 (numérique)

Découvrir la revue

\section{Citer cet article}

Downs, C. (2001). Mythe cosmogonique et dialogue scientifico-religieux.

Théologiques, 9(1), 95-111. https://doi.org/10.7202/005685ar

\section{Résumé de l'article}

S'inspirant de la Dogmatique de Gérard Siegwalt, la présente se veut une réaction face à l'acosmisme théologique contemporain par le biais d'un questionnement touchant certaines de ses positions caractéristiques, en l'occurrence celles formulées par le groupe de l'Institut de recherches herméneutiques et systématiques de l'université de Neuchâtel dans le cadre d'une actualisation du projet bultmannien de démythologisation. Il ne s'agit pas ici d'évaluer la pertinence de ce projet qui insiste à juste titre sur le concept théorique d'existence, mais simplement de se pencher sur l'attitude intransigeante qu'il adopte face aux questions cosmologiques, et spécialement face à l'antique cosmogonie mythique. On cherchera ainsi à aller à l'encontre d'une approche réductrice du mythe cosmogonique qui ne voudrait voir en lui qu'une représentation purement fictive du monde " devenue obsolète ". 


\section{Mythe cosmogonique et dialogue scientifico-religieux}

Christian Downs

Faculté de théologie Université de Montréal

S'inspirant de la Dogmatique de Gérard Siegwalt, la présente se veut une réaction face à l'acosmisme théologique contemporain par le biais d'un questionnement touchant certaines de ses positions caractéristiques, en l'occurrence celles formulées par le groupe de l'Institut de recherches herméneutiques et systématiques de l'université de Neuchâtel dans le cadre d'une actualisation du projet bultmannien de démythologisation. Il ne s'agit pas ici d'évaluer la pertinence de ce projet qui insiste à juste titre sur le concept théorique d'existence, mais simplement de se pencher sur l'attitude intransigeante qu'il adopte face aux questions cosmologiques, et spécialement face à l'antique cosmogonie mythique. On cherchera ainsi à aller à l'encontre d'une approche réductrice du mythe cosmogonique qui ne voudrait voir en lui qu'une représentation purement fictive du monde « devenue obsolète ».

\section{Présentation du problème}

Siegwalt ${ }^{1}$ fait remarquer que deux attitudes prévalent encore aujourd'hui, en réaction à l'infirmation des représentations cosmologiques mythiques traditionnelles par les observations de la science

1. Gérard SIEGWALT, Dogmatique pour la catholicité évangélique. Système mystagogique de la foi chrétienne, Genève, Paris, Labor et Fides, 1986-2000. Tome I. Les fondements de la foi. Vol. 1. La quête des fondements. Vol. 2. Réalité et révélation. Tome II. La réalisation de la foi. Vol. 1. L'Église chrétienne dans la société humaine. Vol. 2. Les médiations : L'Église et les moyens de grâce. Tome III. L'affirmation de la foi. Vol. 1 Cosmologie théologique: Sciences et philosophie de la nature. Vol. 2. Cosmologie théologique: Théologie de la création. Deux autres tomes 
moderne ${ }^{2}$. La première consiste à rejeter l'image religieuse du monde dans sa totalité. La seconde cherche à départager au sein de cette image ce qui est proprement religieux de ce qui est pré-scientifique caduque $^{3}$. Le projet de démythologisation opte pour la seconde attitude. Son objectif est d'actualiser l'essence du message biblique afin qu'il devienne compatible avec l'image moderne du monde. Or, le "kérygme biblique se présente sous la forme d'un mythe, non seulement à propos des puissances cosmiques, mais aussi à propos de $\mathrm{Dieu}^{4}$ ». Dans l'esprit du projet bultmannien, que cela soit au niveau des puissances ou de Dieu, l'un et l'autre relèvent de la mythologie; l'un et l'autre consistent à objectiver ce qui ne peut être objet : " La transcendance n'est pas objective-extérieure, elle est subjectiveexistentiale ${ }^{5} »$. Nous reviendrons sur ce point décisif ultérieurement. Contentons-nous pour le moment de citer la position du groupe de recherche de l'Institut ${ }^{6}$ à l'égard de la mythologie cosmogonique, puis de présenter les grandes lignes de son projet.

a) Dans son essence, la mythologie constitue une vision dépassée du monde et, si la foi consiste à la tenir pour vraie aujourd'hui, alors la foi n'a plus de validité, et il faut l'abandonner. La foi ne garde une chance que si on peut dépouiller son message de la gangue mythologique.

b) Cela ne veut pas dire qu'il faille à tout jamais renoncer à utiliser des expressions mythologiques. Bultmann ne prévoit pas de rééditer les Écritures en jargon existential. La référence demeure le nouveau testament ; c'est lui qu'on lit, dans son langage et avec ses représentations mythologiques; mais celles-ci n'auront alors plus qu'une valeur symbolique, métaphori-

portant sur L'affirmation de la foi suivront : une Anthropologie théologique et une Théologie théologique empruntant la même approche systématique en deux volumes. Il ne sera pas question ici de synthétiser le contenu de cette dogmatique, mais seulement de s'y référer - le plus directement possible - à l'occasion d'une réflexion dont nous portons l'entière responsabilité.

2. À savoir que les dieux, ou puissances, ne régissent pas les lois de la nature et ne doivent conséquemment pas leur être identifiés.

3. SIEGWALT, III/1, p. 142.

4. SIEGWALT, III/2, p. 257.

5. SIEGWALT, III/2, p. 257.

6. Pierre BÜHLER et Clairette KARAKASH (éd.), Science et foi font système, une approche herméneutique, Genève, Labor et Fides, 1992, 211 p. À noter que notre critique ne touche que la première partie de cet ouvrage qui porte sur la mise au point d'un modèle théorique. 
que, spirituelle, etc., par la force des choses (décalage entre l'univers antique et le nôtre).

...On peut imaginer le monde générique (originaire) de la mythologie comme uni et homogène, indistinct ; appelons-le l'idéal mythologique et concevons qu'il soit avant tout et largement fictif?.

Le projet de l'Institut vise à poser les assises d'un dialogue impliquant une herméneutique bultmannienne démythologisante et antimétaphysique de l'expérience " ordinaire interhumaine, de la quotidienneté et de l'immédiateté ${ }^{8}$ ", qui cherche à définir les structures fondamentales de la subjectivité existentielle, soit l'existential, et qui représente la démarche proprement théologique penchée sur la foi, et une herméneutique de la démarche scientifique contemporaine qui valorise l'attitude anti-déterministe que l'émergence du concept d'imprévisibilité a permis d'adopter'. Ce concept, ainsi que le nouveau paradigme au sein duquel il opère, tous deux étrangers à la perspective de Bultmann, imposent une conception de la science qui n'est plus celle d'une rationalité analytique absolutisée, cherchant à décomposer le réel en éléments simples, mais plutôt celle d'un effort jamais achevé de la raison en constante confrontation avec le réel ; une raison opérant au sein d'un système ouvert d'interactions et dirigée vers l'affûtement d'une approximation infinie ${ }^{10}$. Aussi, la foi, de son côté, qui consiste en une conviction produite à l'occasion d'une « expérience fondatrice de

7. Pierre-Luigi DUBIED, "Imprévisible et réel commun ", dans BÜHLER et KARAKASH (éd.), Science et foi, p. 119-120.

8. Clairette KARAKASH et Pierre BÜHLER, "Introduction », dans BÜHLER et KARAKASH (éd.), Science et foi, p. 39.

9. La systémique stipule l'existence d'un principe néguentropique qui fait en sorte que le degré de désordre ou entropie, relatif à un système ouvert (en interaction avec le milieu) ne va pas dans le sens d'un accroissement continu (tendance vers l'énergie minimum), mais bifurque de manière imprévisible vers de nouvelles zones d'équilibre. Ce passage à un état d'organisation plus complexe se manifeste sous l'influence de ce que I. Prigogine a nommé : les structures dissipatives. L'apparition de telles structures ne peut être prédite à partir des données initiales du système pour des raisons qui dépassent la simple inacuité de nos observations; on parle ici d'une irréversibilité ontologique et non plus seulement théorique/méthodologique.

10. Pierre BüHLER, "Faille, conformité ou dialogue critique? Une approche systémique des interactions entre la foi et la raison ", dans ibid, 
révélation, d'illumination ou de libération (buisson ardent, croix, etc.) » 11 , doit éviter de s'absolutiser pour devenir un point de vue doctrinaire ; son système de convictions, fruit d'une herméneutique de l'expérience fondatrice, doit également demeurer ouvert. Dans un tel contexte, les chercheurs scientifiques doivent perdre leur "naïveté théologique » et ne pas franchir les limites inhérentes à leur discipline ${ }^{12}$. De leur côté, il faut que les théologiens « vulgarisent le chemin parcouru, qu'ils expliquent aux scientifiques pourquoi et comment Dieu a changé de statut ${ }^{13}$. Dans la mesure où science et théologie se présentent comme des efforts interprétatifs, l'un empruntant les modalités d'une connaissance empirico-formelle falsificationiste et intersubjective du réel, l'autre se penchant sur la foi en tant qu'expérience subjective existentiale, il faut tenter de dégager une approche systémique qui puisse chapeauter sur le plan épistémologique le dialogue entre les deux, un système qui permette d'articuler avec prudence les niveaux respectifs de ces différents types de discours, et de faire valoir la complémentarité dans la différence ${ }^{14}$.

\section{Du dualisme méthodologique au dualisme ontologique et vice versa}

On voit bien en quoi une telle démarche est, somme toute, conforme au projet bultmannien de démythologisation et manifeste à n'en pas douter un certain acosmisme théologique. C'est un cas typique de compartimentage ${ }^{15}$ qui, s'il n'hérite pas directement du projet de la mathesis universalis, apparaît pour le moins très familier à la distinc-

p. 75 ; « Au travers des révolutions relativiste, quantique, cybernétique, thermodynamique et biologique (biologie moléculaire, génétique, écologie), le rapport des scientifiques aux objets qu'ils étudient a profondément changé ; du jeu s'est introduit entre leurs théories et la réalité qu'elles appréhendent. Du coup le réel s'est voilé, obligeant les savants à s'interroger sur les fondements de la science, sur le statut de la connaissance. » P. BÜHLER, C. KARAKASH, op. cit., p. 28.

11. BÜHLER, "Faille, conformité ", p. 76.

12. BÜHLER et KARAKASH, "Introduction », p. 32.

13. Ibid, p. 33.

14. Ibid, p. 36 .

15. Ted PETERS emploie l'expression «two language theory ": "Theology and Science : Where are We ? ", in : Zygon, Vol. 31, No. 2, 1996, pp. 323-343: 
tion cartésienne opérée entre res extensa et res $\operatorname{cogitans}^{16}$. Une telle démarche est marquée par le dualisme ontologique. Elle opère une scission entre l'esprit et la matière, entre l'anthropologie et la cosmologie, entre la sotériologie et la théologie de la création. Selon Siegwalt, le rapprochement entre ces pôles doit s'effectuer par un dépassement du dualisme, c'est-à-dire non dans le compartimentage, le réductionnisme, le monisme matérialiste ou spiritualiste, mais plutôt dans le sens d'un holisme dialectique où l'unité du monde est posée mais vécue dans sa diversité et dialectiquement.

Le schéma sujet-objet, homme-monde, est constitutif de l'être-au-monde de l'homme historique. Il définit la base même du rapport de l'homme au monde. Il n'est cependant pas seul à le faire, car il s'inscrit à l'intérieur d'une participation fondamentale de l'homme au monde qui est par-là son milieu. Aussi le rapport sujet-objet n'est-il pas par luimême déjà dualiste : il exprime au contraire une dualité (qui est différente du dualisme) à l'intérieur d'une unité dialectique ; l'unité est différenciée, elle est à proprement parler une unité duelle. Il y a dualisme quand le schéma sujet-objet est absolutisé, quand il est coupé du rapport de participation de l'homme au monde dans lequel il s'inscrit ${ }^{17}$.

D'une façon analogue à Jung, Siegwalt conçoit la matière et la psyché comme les deux pôles d'une même réalité qui les transcende. Il rejette d'entrée de jeu l'absolutisation méthodologique et ontologique du dualisme scientifique en alléguant que les énoncés qui en sont issus peuvent bien être appréciés comme étant justes, il n'en demeure pas moins qu'ils ne sont pas pour autant vrais. À cet égard, il cite à

"A problem I have with the two-language theory is that it gains peace through separation, by establishing a demilitarized zone that prevents communication. In the event that a scientist might desire to speak about divine matters or that a theologian might desire to speak about the actual world created by God, the two would have to speak past one another on the assumption that shared understanding is impossible. Why begin with such an assumption? »

16. Dans le cadre des philosophies idéalistes allemandes postérieures au cartésianisme (Kant et Fichte), ce dualisme, hérité de la tradition néoplatonicienne, consistera à voir la nature comme ce qui est essentiellement non-moi (Nicht-Ich). Également en relation avec cette tradition, il faut mentionner l'influence de la philosophie patristique qui a établi un lien étroit entre péché et nature. SIEGWALT, III/1, p.145.

17. Siegwalt I/2, p.24. 
plusieurs occasions la phrase du philosophe théologien Georg Picht : "Une science qui détruit la nature ne peut être vraie ${ }^{18}$."

Bien qu'elles se manifestent dans des dynamiques de compréhension toujours renouvelées, théologie et connaissance scientifique ne permettent respectivement de se prononcer sur le réel que dans des perspectives parallèles. Elles ne peuvent, à l'intérieur des limites qu'elles se sont imposées, parvenir à une vision unifiée de la réalité où l'être humain serait partie intégrante de son milieu, où existerait non seulement une continuité, mais bien une complémentarité ontologique entre les plans de l'extériorité et de l'intériorité. Un tel champ dialogique semble nous laisser en présence d'un vide ontique. Il situe l'homme, être spirituel, au milieu d'un monde déshumanisé, voir aseptisé à grands coups d'abstractions mathématiques ${ }^{19}$. Il se limite à un mode de connaissance dualiste, fonctionnaliste et partiale de la

18. Der Begriff der Natur und seine Geschichte, Stuttgart, Klett-Cotta, 1989, p.10. Cité par Siegwalt dans «Science de la nature et théologie de la création ", Théologiques, 7/1, Montréal, 1999, p.80.

19. Si on suit la définition de Jean LADRIÈRE (L'articulation $d u$ sens, vol. I, Paris, Cerf, 1984 (1970), p. 25-50), les sciences empirico-formelles se rapportent à l'expérience empirique à l'aide d'un appareil théorique hérité des sciences formelles. Le langage théorique des sciences empirico-formelles contient des outils logiques et mathématiques plus puissants que ceux du langage empirique. Les propositions vraies (axiomatiques) le sont à titre d'hypothèses. "Moyennant l'intervention de règles de déduction acceptées par la théorie ", on peut en déduire des théorèmes. (p. 32)

"Grâce à l'observation on enregistre l'état du système étudié à un instant déterminé ; autrement dit, on se donne les conditions initiales du problème traité. Ces conditions sont exprimées dans une proposition empirique dont le contenu, grâce aux règles de correspondances, est injecté dans le langage théorique sous la forme d'une certaine proposition théorique. Cette dernière proposition, jointe aux axiomes de la théorie, permet d'obtenir, grâce aux règles de déduction, une nouvelle proposition que l'on pourra traduire dans le langage empirique sous la forme d'une proposition exprimant un certain état de choses relatif au système étudié à un moment donné (postérieur à l'instant auquel correspondent les conditions initiales). Si cet état de choses est effectivement celui qui est observé à cet instant, on en a fourni une explication. $»($ p. 33)

Mais à quoi réfèrent les signes ? Le point de vue réductionniste lié à l'empirisme strict ramène tout le langage scientifique aux propositions 
nature. Il réduit celle-ci à un réseau discontinu des lois formelles mises à jour dans des conditions techniques/instrumentales d'expérimentations ultra spécifiques où seule prévaut la question du " comment»; celles du «quoi », du "pourquoi » (origine) et du « en vue de quoi » (fin) étant en bout de ligne laissées pour compte.

Le concept dominant de science est celui d'une science réductrice : se concevant comme purement inductive, c'est-à-dire s'appuyant sur les seuls faits " objectivement " avérés, elle limite en fait ces derniers à ceux qui correspondent au schéma dualiste et à une conception largement " positiviste " de l'objectivité, à l'exclusion des autres niveaux (psychique, paraphysique et parapsychique) et de toute la dimension invisible de la réalité ; l'induction dans ce sens est une réduction. Le vrai concept de science non seulement est ouvert aux différents niveaux de la raison et de la réalité, ouvert également par-delà la dimension visible à la dimension invisible des choses, mais encore est attentif, outre à la totalité de la raison et de la réalité, à leur dimension dernière, ontologique. La raison ontologique pose en effet la question de l'Être comme fondement (et fin) de la réalitée ${ }^{20}$.

« observationnelles ». Or, ces dernières n'expriment pas directement le donné observé « mais plutôt la façon dont ce donné perceptif est interprété » (p. 37). L'observation n'est pas indépendante de la théorie mais bien conditionnée par celle-ci. C'est là le « cercle méthodologique des sciences empirico-formelles ». (p. 38) «La théorie n'est pas une image du monde, c'est seulement une reconstruction conjecturale de la réalité. » (p. 39)

Cela nous permet de mieux comprendre Siegwalt lorsqu'il dit : « C'est que les lois sont des abstractions, et cela doublement : d'une part, elles disent des généralités d'ordre statistique alors que le réel, tout en "obéissant" à ces lois, est bien plus complexe que cela, d'autre part, elles isolent dans le réel tels aspects au détriment d'autres aspects, déconstruisant par conséquent le réel. Non pas que les lois ne seraient pas justes, mais coupant d'un côté le comment du pourquoi et du en vue de quoi, décomposant de l'autre côté le réel ainsi réduit à sa fonctionnalité, elles donnent, là où elles sont absolutisées, comme c'est la tendance dans la civilisation moderne et contemporaine, l'illusion de rendre compte du réel en vérité alors qu'elles ne disent le réel que de manière partielle ; les sciences de la nature sont ainsi partiales. » III/1, p. 231.

20. Siegwalt I/2, p. 91. 


\section{Non-séparabilité, holisme dialectique et affirmations métaphysiques}

L'acosmisme théologique de l'époque contemporaine est lié à un découragement face à l'incommensurabilité entre modèle mécanique et représentation mythique du cosmos, découragement qui a mené à l'acceptation de l'idée que " les affirmations dernières, dites métaphysiques dans le langage traditionnel, ne relèvent pas d'une démarche de connaissance scientifique qui pourrait se situer en continuité directe avec l'effort scientifique ${ }^{21} »$. Il va de soi que la science, en tant que discipline empirico-formelle ne peut pas se prononcer sur des réalités situées au-delà de son champ d'expérimentation. Il va de soi également que la métaphysique, dans le sens traditionnel, ne relève pas d'une démarche empruntant le même appareillage épistémologique que les sciences empirico-formelles; le type d'expérience auquel elle réfère, son mode "d'expérimentation", n'implique pas les mêmes facultés de connaissance. Il reste que science et métaphysique formulent des énoncés qui renvoient à des expériences pensées. L'histoire des sciences empirico-formelles comme celle de la métaphysique, dont les origines remontent à la protohistoire et à la préhistoire, partagent un héritage commun : dans l'une et l'autre, "la pensée est enracinée dans l'expérience de l'échec ${ }^{22}$ "; pour l'une et l'autre, quoi qu'en pensent certains physiciens, la question de la vérité n'a pas de réponse définitive : elle se présente plutôt comme une quête perpétuelle.

La pensée est ainsi essentiellement symbolique : elle vise au-delà d'ellemême, vers la vérité qui est la totalité consciente d'elle-même, et néanmoins elle est elle-même déjà transparente à cette totalité, riche d'elle, une expression relative de cette totalité. La pensée est toujours une pensée historique, la pensée d'un homme donné, fondée dans une expérience donnée. Mais cette pensée sera toujours, précisément parce que symbolique, une pensée typique, dans toute sa relativité, c'est-à-dire que sa particularité contiendra toujours une universalité qui est celle de la véritée ${ }^{3}$.

Sans vouloir tomber dans un débat de fond et au risque de paraître naiff, nous comprenons mal pourquoi la possibilité même d'une certaine continuité, toujours à refaire, entre énoncés scientifiques et énoncés

21. Siegwalt, BÜHLER et KaraKaSH, "Introduction », p. 40.

22. SIEGWALT, III/1, p. 138.

23. SIEGWALT, III/1, p. 139. 
métaphysiques est rejetée du revers de la main, en quelque sorte $a$ priori. Les théories de la physique contemporaine, outre le fait qu'elles vont à l'encontre du déterminisme, ont également bouleversé les notions classiques de temps, d'espace et de matière. Maintenant, on parle en tout d'espace-temps et d'énergie-matière, si bien que, si d'un certain point de vue, l'univers peut apparaître comme une immensité effarante, d'un autre, il n'est pas impossible de l'imaginer tel un champ virtuel ne possédant aucune réelle extension. On doit insister sur ce point dans la mesure où le problème du dualisme ontologique, qui est celui de la relation entre matière et psyché, entre kosmos et anthropos, a vu le jour dans un épistémè où régnait une compréhension euclidienne géométrique (longueur/largeur/profondeur) et newtonienne mécanique (relations entre force, masse, mouvement et vitesse) de l'espace et du temps. La modernité $a$, en effet, développé la thèse du dualisme ontologique dans le cadre d'une compréhension de l'espace et, corrélativement, de la nature dans son ensemble, en tant que chose étendue (res extensa), " comme une réalité essentiellement extérieure ${ }^{24}$ ». Chez Descartes et Newton, l'espace est absolu, homogène et isotrope : « il est le contenant dans lequel il y a tels contenus mesurables donnés ${ }^{25}$ ". Dépassant le dualisme classique, Kant abordera la notion d'espace absolu de Newton dans le contexte de son esthétique transcendantale, l'espace n'étant pas pour lui "simplement empirique au sens d'extérieur, mais aussi fondamentalement principiel et donc intérieur ${ }^{26}{ }$.

À l'époque contemporaine, le schéma dualiste sujet-objet et par conséquent la conception dualiste de l'espace cèdent la place à un espace noneuclidien, du fait de la quatrième dimension du temps (l'espace, c'est l'espace-temps) qui implique l'affirmation de la courbure de l'espace (cf. la théorie de la relativité d'A. Einstein); et ils cèdent aussi la place à un espace non-newtonien, à cause de l'équivalence de la matière et de l'énergie (dans la physique quantique) et de la théorie systémique du ou des champs de force qui y est liée. S'il est vrai que la géométrie euclidienne reste valable pour les corps rigides et Newton au plan de l'expérience moyenne, ils ne s'appliquent pas au plan de l'infiniment petit ni à celui de l'infiniment grand [...]. [L]e concept d'espace est celui d'un continuum spatio-temporel dont nous avons dit ci-dessus qu'il concerne tout, de

24. SIEGWALT, III/1, p. 264.

25. Ibid, p. 264.

26. Ibid, p. 265. 
l'énergie/matière au vivant et même au psychique. Le monde est un champ de forces ou un ensemble de champs de forces multiples relevant d'un même champ de forces. C'est l'affirmation d'un monde dialectiquement unitaire, fait de relations entre toutes ses parties ${ }^{27}$.

Une telle théorisation de l'espace à l'époque contemporaine, bien qu'elle aille dans le sens de la dématérialisation, d'une déconcrétisation moins fallacieuse du cosmos, demeure empirico-formelle fonctionnaliste et donc réductrice. Elle ne concerne que le comment de l'espace tel qu'il se manifeste à nos instruments aux échelles microcosmique (quantique), macrocosmique (relativiste) et mégacosmique (euclidien/newtonien) et ne répond pas à la question de son essence. Aussi, bien que la science admette la non-séparabilité à l'échelle cosmique sur le plan physique, elle ne cesse pas pour autant de dresser un portrait du réel marqué par une série de discontinuités : celles entre énergie/matière (inorganique, inanimée) et bios (organique, animé), entre bios et psychè, entre kosmos et anthropos ${ }^{28}$. Ces discontinuités, qui, notons-le, concernent les relations entre certaines des données élémentaires mythiques symboliques de la nature, sont pour la science autant d'apories à résoudre et traduisent, nous dit Siegwalt, l'impossibilité dans laquelle elle se trouve à poser des jugements d'ordre métaphysique. Cela, d'une façon d'autant plus flagrante que la science tend également à postuler l'existence d'une certaine continuité du cosmos dans des théories comme le big-bang (cosmogenèse), l'évolutionnisme (phylogenèse), la systémique, l'écologisme, le principe anthropique, l'autopoïèse, etc.

Par l'évidence à la fois de la continuité et de la discontinuité du réel, ce qui revient à poser la question du fondement de cette polarité et aussi celle de sa fin, et ce à propos tant de l'univers en général que de l'homme en particulier, la physique contemporaine est marquée par une incertitude dernière qu'il n'est pas en son pouvoir, en tant que physique, de dépasser, car elle est d'ordre métaphysique. Mais la non-séparabilité affirmée au plan physique et qui s'ouvre aussi au plan biologique, et jusqu'au plan de l'esprit, implique, par-là même, celle du physique et du métaphysique. C'est pourquoi il ne peut être question de la physique en soi et pour soi $^{29}$.

27. SIEGWALT, III/1, p. 265.

28. Ibid, 80 ss.

29. Ibid, p. 91. 
N'est-il donc pas possible d'actualiser le mythe afin que la foi qui le tiendrait pour vrai garde sa validité ? N'existe-t-il pas des raisons qui pourraient contribuer à justifier l'antique description mythique symbolique du cosmos ? Autrement dit, dans quelle mesure n'est-il pas possible, à la lumière d'une raison qui se risque certes, mais qui tend vers une plus grande intégration du divers de l'expérience, de mettre à jour une pensée qui permettrait de rendre plausible une compréhension mythique symbolique du cosmos?

\section{Philosophie de la nature et pensée mythique}

La conception de la science de la nature mise de l'avant par Siegwalt - à comprendre dans le sens d'une philosophie de la nature (Naturwissenschaft) - se veut le fruit d'une pensée totalisante - et non totalitaire - qui relie (religare) la partie au tout, qui recueille et rassemble (relegere) la partie et le tout ${ }^{30}$. Ce faisant, elle ne peut qu'ouvrir à la dimension de transcendance de la totalité, d'où son caractère mystagogique. Ainsi, la science religieuse et mystagogique de la nature ${ }^{31}$ pose, outre la question du "comment ", celles du « quoi », du « pourquoi » (origine) et du « en vue de quoi » (fin). Elle ne fonde pas uniquement sa pensée sur les données de l'expérimenta-

30. G. SIEGWALt, « La justesse fonctionnelle de la science et la question de la vérité ", dans Revue d'histoire et de philosophie religieuse, vol. 74, 1994/ 3 , p. 253, note 10 .

31. À cet égard, il faut mentionner que conformément à l'approche systématique de la Dogmatique pour la catholicité évangélique, le premier volume de la Cosmologie théologique, qui porte le sous-titre Sciences et philosophie de la nature, adopte la démarche sapientiale ascendante fondée dans la raison ontologique et prépare la deuxième partie, consacrée à la Théologie de la création, qui elle, adopte la démarche prophétique descendante fondée sur la foi. Cette première partie est, à proprement parler, une démarche pré-dogmatique qui ne part pas de la foi - en tant que révélation spéciale extrinsèque - pour aller au monde, mais à l'inverse va du monde à la foi - en tant que révélation universelle intrinsèque. Bien que la dimension personnelle de la révélation spéciale lui concède un pouvoir plus fondamental de récapitulation du sens dernier des choses, elle ne pourrait pas avoir lieu si préalablement l'homme n'était pas déjà en attente dans le monde. Sur la relation entre démarche sapientiale et démarche prophétique, voir : J.P. GABUS, "La dogmatique de Gérard Siegwalt ", dans Nouvelle revue théologique, 119, 1997, pp. 559-572. 
tion instrumentale des phénomènes (experimentum), mais tend à considérer la diversité des facettes de notre expérience de la réalité (experientia). La science religieuse se refuse en somme à absolutiser les énoncés ou les fondements de toute raison formelle partielle, sans pour autant déprécier leur valeur de vérité. Elle demeure ouverte à l'ensemble des réalités accessibles à la raison, c'est-à-dire au " cœur et à ses fonctions ". En dernière instance, elle devient une philosophie ontologique de l'Être - l'Être comme principe et fin de ce qui est (les étants) - qui doit se constituer conjointement à une réactualisation de la pensée mythique symbolique (cosmogonie, théogonie).

... cette approche globale, d'une part, est inclusive de l'approche scientifique de la nature complétée par les sciences de l'homme, d'autre part, vit d'une appréhension du réel qui est pré-scientifique, ou autrement scientifique, celle du mythe... S'il est vrai qu'il y a une évolution du " mythe à la raison » (cf. A. Comte), la philosophie ontologique devient stérile et se perd sans le mythe. On peut dire tout à la fois que " le mythe est solidaire de l'ontologie » et que l'ontologie est solidaire du mythe elle est l'explicitation et la clarification dans le sens de la récapitulation, de la substance mythique au plan de la raison ontologique, ou de l'esprit ${ }^{32}$.

Or, comment expliciter et clarifier, dans le sens de la récapitulation, la substance mythique au sein d'une pensée globalisante qui se voudrait inclusive de l'approche scientifique de la nature ? Selon le groupe de l'Institut, le mythe cosmogonique agirait pour " clôturer un monde à l'ouverture inquiétante, il procéderait alors à des objectivations fautives $^{33}$ ». La science, pour sa part, procéderait par objectivation adéquate. "On doit, par conséquent distinguer entre objectivation fautive et objectivation adéquate. L'objectivation fautive consiste à traiter comme objet ce qui n'est pas ou ne peut pas être objet ${ }^{34}$ ». Selon l'esprit du projet de démythologisation toute objectivation de la transcendance porte à faux; on ne peut légitimement, comme le soutient Bultmann, parler à la manière du monde de ce qui n'est pas de ce monde. La transcendance n'est pas objective-extérieure, elle est subjective-existentiale.

32. Siegwalt, III/1, p.194.

33. DubIED, "Imprévisible et réel commun », p. 120.

34. Ibid, p. 120. 
Outre le fait que l'objectivité fautive soit fondée sur le dualisme et mette de l'avant une conception plutôt stricte de « l'objectivité » relativement fermée aux déterminations qui dépassent l'horizon de la conscience de veille, elle refuse de créditer la profondeur symbolique des énoncés mythiques relatifs à la cosmologie autrement que dans une perspective intérieure-existentiale. De plus, elle confond la transcendance relative et ses degrés avec la transcendance absolue. Il faut, dans l'esprit de la science religieuse mystagogique de la nature, tenter de développer une approche qui situe davantage les degrés de la transcendance (relative) et qui tente, autant que faire se peut, de marquer l'horizon audelà duquel elle acquiert véritablement le statut de transcendance (relative). Ainsi, en traitant les questions de la réalité et du pouvoir d'éventuels facteurs transcendants, nous adoptons, avec Siegwalt, une approche dialectique unitaire globale où la raison ne serait pas fermée aux différents niveaux d'elle-même et de la réalité. De la sorte, si le psychique est invisible par rapport au physique et au biologique, et que le psychique profond (subconscient et inconscient) demeure le plus souvent invisible au psychique superficiel, ces domaines, qui constituent les objets phénoménologiques de la psychologie des profondeurs, demeurent relativement visibles; ils ne doivent pas être identifiés au domaine proprement invisible, parapsychique et paraphysique. Toutefois, nous dit Siegwalt, il ne faudrait pas confondre la transcendance invisible avec la transcendance de Dieu, le ciel des anges avec le ciel de Dieu, le premier est relatif à la création visible, le deuxième est absolu.

Celui-ci concerne la dimension dernière de l'espace, alors que la dimension invisible de l'espace est avant dernière. Elle relève du créé ; elle est transcendante relativement : elle est la face du réel qui, par rapport à la face immanente, est transcendante. Par rapport à la transcendance à proprement parler, elle relève de l'immanence qui a les deux dimensions visible et invisible et, dans ce sens relatif, immanente (mesurable) et transcendante (non mesurable). On peut désigner les deux dimensions aussi en parlant de la face extérieure et intérieure du réel et, partant, de l'espace, étant entendu que là encore, concernant la face intérieure, il faut distinguer entre les deux acceptions de l'intériorité : l'intériorité ontologique, dernière, et l'intériorité avant dernière ; c'est celle qui est désignée par la dimension invisible du réel. Quel est cet espace invisible? C'est l'espace du mythe $e^{35}$.

35. SIEGWALT, III/1, p. 272. 
Le mythe devient ainsi plus qu'une image symbolique (au sens faible) dont on pourrait abstraire le sens dans une perspective subjective existentiale. Il possède plus qu'une valeur subjective "symbolique, métaphorique, spirituel, etc. ${ }^{36}{ }^{\prime}$. Il traduit un dessein anagogique qui concerne aussi bien l'intériorité que l'extériorité du réel, aussi bien l'espace intérieur que l'espace extérieur. Le mythe, entendons «le monde générique (originaire) de la mythologie ${ }^{37}$ ", parce qu'il définit la nature même de l'espace invisible, devient fondement de l'espace visible. "Si C. G. Jung peut dire : "est réel ce qui est effectif (ist wirklich was wirkt)", la réalité invisible, transcendante, est bien alors réelle ; elle s'atteste dans le pouvoir qu'elle exerce sur l'homme ${ }^{38}$. "

À cet égard, penchons-nous, sur un passage d'une épître paulinienne relatif à la mythologie cosmogonique antique : "En lui (le Christ) ont été créées toutes les choses qui sont dans les cieux et sur la terre, les visibles et les invisibles, trônes, seigneuries, principautés, autorités. »(Col. 1, 15 ss.). Siegwalt s'interroge :

Est-il concevable que ce théologien missionnaire, formé dans le rabbinat juif, connaissant la dure réalité de la loi, ait parlé dans des termes semblables de ces rudiments et de ces puissances s'il n'y avait vu que superstitions, s'il n'avait pas éprouvé la réalité de ces puissances ? Qu'il en ait parlé dans le langage gnostique de l'époque, c'est évident, mais le caractère peut-être désuet de ce langage ne peut nous dispenser de poser la question de la réalité qui correspond à ces rudiments. Que pour Paul ils aient été des puissances astrales se traduisant dans un certain nombre de prescriptions concernant la vie naturelle de l'homme, le manger et le boire et les fêtes qui scandent le cycle des temps, cela tombe sous le sens ;

36. DUBIED, « Imprévisible et réel commun » p. 119.

37. Ibid. p. 120.

38. SIEGWALT, III/2, p. 264. Précisons que dans le cadre de la psychologie jungienne, les archétypes sont définis comme des structures de connaissances et d'actions qui trouvent leur matière dans l'expérience humaine. Les archétypes sont en eux-mêmes de nature métapsychique ou psychoïde, c'est-àdire qu'ils ne peuvent être appréhendés qu'indirectement au travers des représentations archétypiques qu'ils suscitent et dont la teneur est essentiellement symbolique. Ces représentations interviennent à tous les niveaux de l'activité humaine et orientent le devenir ontogénétique ; ambivalentes, elles peuvent être destructrices ou constructrices dépendamment du caractère de l'individu, de ses dispositions naturelles et de l'attitude responsable ou irresponsable qu'il adopte vis-à-vis elles. 
mais à quoi se mesurait pour Paul la réalité de ces puissances : à leur qualité d'astres ou au pouvoir que les prescriptions, les doctrines et ordonnances des hommes (Col 2, 20-22) exerçaient sur les hommes ${ }^{39}$ ?

La question ici n'est donc pas celle de l'actualité des préceptes et ordonnances évoqués, qui, de toute évidence, ne peuvent plus s'appliquer tels quels à notre époque, mais bien celle du pouvoir effectif de ces préceptes, celle de l'enjeu qui s'y joue et celle de la réalité à laquelle ils renvoient. Siegwalt soutient qu'en traitant de ces questions, Paul emprunte la double démarche sapientiale et prophétique (voir note 31). D'une part, il induit, à partir d'observations touchant la loi en tant qu'ordre institué des hommes, "que là advient quelque chose qui dépasse cet ordre : l'homme est "sous la loi » (bypo nomon, Ga 3, $23 ; 4,5)^{40}$ ». C'est-à-dire qu'il pose les réalités angéliques et démoniaques comme puissances constructrices et destructrices appartenant à la création invisible conçue comme une réalité (relativement) transcendante se situant dans un rapport de continuité avec l'immanence - soit la création visible - et soutenant celle-ci. D'autre part, empruntant la démarche prophétique, il éclaire l'enjeu du combat entre ces puissances à la lumière de la révélation spéciale de Dieu à Israël et en Jésus Christ ${ }^{41}$.

\section{Conclusion}

Concluons simplement en disant que chez les anciens, la question de la réalité des puissances angéliques et démoniaques était associée au monde astral, céleste, supra-lunaire, etc., avec toutes les subtilités phénoménologiques et la complexité épistémologique que ces conceptions impliquent. Par ailleurs, si la psychologie contemporaine semble

39. Siegwalt, III/2, p. 258

40. Ibid, p. 262.

41. "Si les puissances angéliques et démoniaques se manifestent dans le pouvoir qu'ont la morale et la législation sur l'homme, le programme de démythologisation de Bultmann porte à faux. Si les puissances et les rudiments sont des réalités mythiques, c'est-à-dire des réalités fondamentales qui affleurent dans la morale et la législation de la société, alors il ne peut s'agir de démythologiser, mais uniquement ceci : montrer quels préceptes, ordonnances et doctrines d'aujourd'hui, quelle morale donc et quelle législation de nos jours sont transparents à des puissances angéliques et démoniaques. C'est là, du point de vue historique et exégétique, la seule vraie question. »Ibid, p. 259. 
situer la transcendance dans les arrières-plans obscurs de la psyché, la notion de synchronicité, en tant que principe de connexion acausal entre les plans psychique et physique, nous force à nuancer cette position. Dans la perspective du holisme dialectique adoptée ici, la question de la réalité de ces puissances ne peut être simplement reléguée à la sphère anthropologique, même comprise collectivement. La réalité invisible n'est pas plus subjective-intérieure qu'elle n'est objectiveextérieure. Elle concerne la totalité du réel, c'est-à-dire aussi bien ses aspects extérieur-objectif, qu'intérieur-subjectif.

Nous l'avons dit : ce pouvoir ne saurait être limité à sa portée anthropologique. Il vaut d'abord pour le cosmos et la nature visibles eux-mêmes, il a donc une portée d'abord cosmologique et alors aussi une portée anthropologique. Si saint-Paul l'établit en référence à l'homme, il n'est pas limité à lui. Et, en tout état de cause, il s'agit là d'une réalité ontique ${ }^{42}$.

En somme, nous soutenons, avec Siegwalt, que l'image hiérarchique du cosmos de l'antique symbolisme mythique doit faire l'objet d'une réflexion sérieuse à une époque où la science et la religion tendent à vouloir renouer leurs liens ; une telle image ne doit pas être interprétée de la part de la théologie et de la philosophie, dans une perspective uniquement subjective-existentiale.

Il n'y a pas de religions vivantes pour qui le ciel de Dieu n'est pas d'une infinie richesse, sans que cela ne mette nécessairement en cause la déité et donc l'unicité de Dieu : si le polythéisme est une tentation constante pour toute religion vivante, le Dieu vivant n'existe pas dans la pureté chimique d'un concept philosophique ou d'une affirmation théologique. Toutes les religions dites monothéistes proclament un Dieu qui est riche de bien des aspects des dieux, lesquels sont récapitulés par lui, et affirment une dimension invisible de la création (le monde des anges). Et toute philosophie qui est une philosophie de la raison totale reconnaît une différenciation ou même une gradation aussi bien dans la dimension visible ( $c f$. les niveaux du réel) que dans la dimension invisible ${ }^{43}$.

42. SIEGWALT, III/2, p. 264.

43. Ibid, p. 218-219. 
RÉSUMÉ

S'inspirant de la Dogmatique de Gérard Siegwalt, la présente se veut une réaction face à l'acosmisme théologique contemporain par le biais d'un questionnement touchant certaines de ses positions caractéristiques, en l'occurrence celles formulées par le groupe de l'Institut de recherches herméneutiques et systématiques de l'université de Neuchâtel dans le cadre d'une actualisation du projet bultmannien de démythologisation. Il ne s'agit pas ici d'évaluer la pertinence de ce projet qui insiste à juste titre sur le concept théorique d'existence, mais simplement de se pencher sur l'attitude intransigeante qu'il adopte face aux questions cosmologiques, et spécialement face à l'antique cosmogonie mythique. On cherchera ainsi à aller à l'encontre d'une approche réductrice du mythe cosmogonique qui ne voudrait voir en lui qu'une représentation purement fictive du monde « devenue obsolète ».

\section{ABSTRACT}

This article is a reaction to the absence of cosmology in contemporary theology. Inspired by Gerard Siegwalt's Dogmatique, it challenges certain positions taken by contemporary theologians, especially those formulated by a group at the Institut de recherches hermeneutiques et systematiques de l'université de Neuschatel. The goal of this group's project is to actualize demythologization based on the work of Bultmann. This article does not evaluate the pertinence of the project itself, but questions its intransigent stance in relation to cosmologies, especially ancient mythic cosmology. It therefore challenges the project's reductive approach that views this mythic cosmology as a purely fictional representation of an "obsolete" world. 